\title{
Cancerous Tissue Diagnosis by LIF Spectroscopy Derived From Body-Compatible Fluorophores
}

\author{
Atefeh Asghari Moghaddam $^{1 \oplus}$, Batool Sajad $^{1 *}{ }^{*}$, Fariba Mehrad Nia $^{\circledR}$, Seyed Hamid Madani ${ }^{\circledR}$ \\ ${ }^{1}$ Physics Department, Alzahra University, Tehran, Iran \\ ${ }^{2}$ Pathology Department, Kermanshah University of Medical Sciences, Kermanshah, Iran
}

*Correspondence to Batool Sajad,

Physics Department, Alzahra University Tehran, Iran Tel: +98- 9123113562 ; Fax: +98-2188047861 Email: bsajad@alzahra.ac.ir

Published online February 27, 2021

\begin{abstract}
Introduction: The laser-induced fluorescence (LIF) method as molecular emission spectroscopy is used to diagnose cancerous tissues. According to the previous reports, the red-shift in the fluorescence spectrum from Rhodamine 6G (Rd6G)-stained cancerous tissues compared to healthy ones impregnated with the same dye provides the feasibility for diagnosis. In this paper, we have employed the LIF emissions as a diagnostic method to distinguish between cancerous and healthy tissues infiltrated by a body-compatible fluorophore to avoid the toxicity and hazard of Rd6G dye. Methods: Biological tissue specimens are stained with sodium fluorescein $(\mathrm{NaFl})$ dye and then irradiated by the blue $\mathrm{CW}$ diode laser $(405 \mathrm{~nm})$ to examine the spectral properties that are effective in detecting cancerous tissues.

Results: The spectral shift and the intensity difference of fluorescence are keys to diagnosing in vitro cancerous breast, colon, and thyroid tissues for clinical applications. The notable tubular densities in the breast and colon tissues and the space between the papillae in the thyroid ones cause the cancerous tissues to be prominently heterogeneous, providing numerous micro-cavities and thus more room for dye molecules.

Conclusion: Here, we have assessed the spectral shift and intensity difference of fluorescence as a diagnostic method to distinguish between cancerous and healthy tissues for clinical applications. Keywords: Laser-induced fluorescence; Diagnosis; Cancerous tissues; Body-compatible dye; Sodium fluorescein.
\end{abstract}

\section{Introduction}

Cancer means the growth, proliferation, and sometimes abnormal release of body cells. All such diseases have an unbridled growth pattern and tend to detach from the source and metastasis. ${ }^{1}$ The prevalence of breast cancer is about one-third of all cancers in women, and it is the most common cause of cancer mortality among women, which significant efforts have been done to diagnose and treat it in a timely manner. Standard methods for diagnosing or preventing breast cancer include $\mathrm{x}$-ray mammography, ultrasonography, magnetic resonance imaging, and positron emission tomography. ${ }^{2}$ Biopsies are usually performed routinely after diagnosing a lesion by various clinical breast examinations. Then the biopsied tissue is fixed in the histology laboratory and stained with special dyes for pathological studies. ${ }^{3}$ Besides, rapid, quantitative, and non-invasive diagnostic methods based on fluorescence spectroscopy, as a link of morphological and biochemical properties of tissues, can be an essential step in the diagnosis and treatment of cancer. ${ }^{4}$ The development of malignancies, in addition to metabolic and structural changes in cell and subcellular levels, can also affect the optical properties of tissue, such as dispersion, absorption, and fluorescence. Therefore, optical spectroscopy contains useful information for the diagnosis of cancerous tumors and is also a promising method for in vitro and in vivo studies of tissues as well as blood. The laser is used as non-ionized coherent radiation to detect the optical properties of soft tissues. ${ }^{5,6}$

In laser-induced fluorescence (LIF) spectroscopy, visible or ultraviolet light is used to stimulate the molecules of the sample. Photons are absorbed in molecular transitions due to Stokes shift (the difference between the maximum absorption line $V_{e x}^{p e a k}$ and the fluorescence radiation $V_{f}^{\text {peak }}$ ) re-emission at longer wavelengths. ${ }^{5}$ Palmer et $\mathrm{al}^{7}$ have evaluated auto-fluorescence spectra derived from healthy and cancerous breast cells and have shown significant differences in their fluorescence properties. According to this report, the cancerous cells gain several receptors as a distinguishable characteristic, unlike healthy cells. Also, and Elshemey ${ }^{8}$ used a $266 \mathrm{~nm}$ diode laser to stimulate healthy, benign, and malignant tissues and produce autofluorescence radiation. It presents differences such as the parameters of the sub-peak range of the fluorescence spectrum and its moderate intensity to distinguish between tissues. Ghasemi et $\mathrm{al}^{9}$ used LIF spectroscopy to 
measure the spectral displacement from stained breast tissue with Rhodamine 6G (Rd6G) to distinguish between healthy and cancerous tissues. They have shown that the fluorescence spectrum derived from cancerous tissues compared to healthy ones move to the longer wavelengths.

In previous reports, tissue samples have been examined by LIF, either by auto-fluorescence or by chemical and toxic Rd6G fluorophores. Braginskaja et al ${ }^{10}$ have also used LIF spectroscopy by injection sodium fluorescein $(\mathrm{NaFl})$ dye into rats to diagnose gastric cancer. They showed that in all cases, due to penetration and the selective accumulation of $\mathrm{NaFl}$ in the tumor, the fluorescence intensity in the tumor stomach tissues was higher than that in the adjacent normal one. In this paper, the spectral shift and the intensity difference of fluorescence were assessed as a diagnostic criterion to detect in vitro cancerous breast, colon, and thyroid tissues for clinical applications. In vitro diagnosis of healthy and malignant tissues using the body-compatible dye through LIF spectroscopy was the purpose of this study.

For the fluorescence diagnosis of human malignancy, $\mathrm{NaFl}$ is permitted and recommended for its high specificity to the tumor. ${ }^{11}$

\section{Materials and Methods \\ Preparation of Samples and Solutions}

Healthy and cancerous in vitro formalin breast, colon, and thyroid tissue samples were investigated in the laboratory. Normal and cancerous tissues were examined from 30 patients affected by three types of cancer-breast, colon, and thyroid. More than 10 healthy and 10 cancerous samples of each kind of tissue were fixed after surgery or biopsy and then cut into pieces $\left(5 \times 3 \times 2 \mathrm{~mm}^{3}\right)$.

Before the LIF spectroscopy examination, the samples were immersed in an optimum concentration (1 mM) of a NaFl solution in ethanol solvent for 3 hours and then exposed to a laser beam. $\mathrm{NaFl}$ is a non-toxic and body-compatible dye in low doses, with a high quantum yield (0.88) and $\mathrm{C}_{20} \mathrm{H}_{10} \mathrm{Na}_{2} \mathrm{O}_{5}$ chemical formula. Unlike healthy breast tissue, which has a high percentage of fat and a yellow appearance, cancerous tissues are stiff and white. The samples are cut into slices with micron size, using a microtome, and then placed on a slide. An optical microscope is also employed to image the paraffinembedded tissues.

\section{Experimental Array}

A blue CW diode laser at a $405 \mathrm{~nm}$ wavelength and $150 \mathrm{~mW}$ power (MLPH-III model/Noor Sanate Pars company) is utilized as an irradiation source to excite the NaFl fluorophores. The LIF emission from the sample was collected by a fiber bundle UV 600 (core)/660 (cladding) type with an SMA-905 fiber connector having NA 0.22 and $1 \mathrm{~m}$ lengths. The fiber output was coupled to the entrance site of a Phystec miniature spectrometer (UVS 2500; Pooyesh Tadbir Karaneh company) over the wavelength range of $200-800 \mathrm{~nm}$ with $0.1 \mathrm{~nm}$ resolution. Figure 1 illustrates the arrangement for LIF experiments.

\section{Results}

First, NaFl solution was prepared in ethanol solvent at various concentrations and exposed to $405 \mathrm{~nm}$ diode laser radiation to obtain the optimal fluorescence conditions. Figure 2 shows the LIF spectrum of $\mathrm{NaFl}$ solutions at different concentrations of $0.1-10 \mathrm{mM}$ to find the optimum conditions.

The fluorescence signal is intensified due to the larger population of molecular transitions. In contrast, the decrease in amplitude arises from static/dynamic quenching, including self-quenching, collisional effects, and the aggregation of monomers to dimers. ${ }^{12}$ So, as shown in Figure 2, the signal magnitude lucidly rises versus the solution concentration (up to $1 \mathrm{mM}$ ), and after that, it decreases with increasing the solution concentration due to the increasing collision effects and Forster resonance energy transfer $\left(K_{t}\right)$ as well as the increase of self-quenching. ${ }^{13-15}$ Therefore, the optimum dose of $1 \mathrm{mM}$ of solution concentrations is considered for staining biological tissues.

Moreover, a significant red-shift took place in the emission by increasing the $\mathrm{NaFl}$ concentration, due to the more re-absorption and re-emission of photons in the

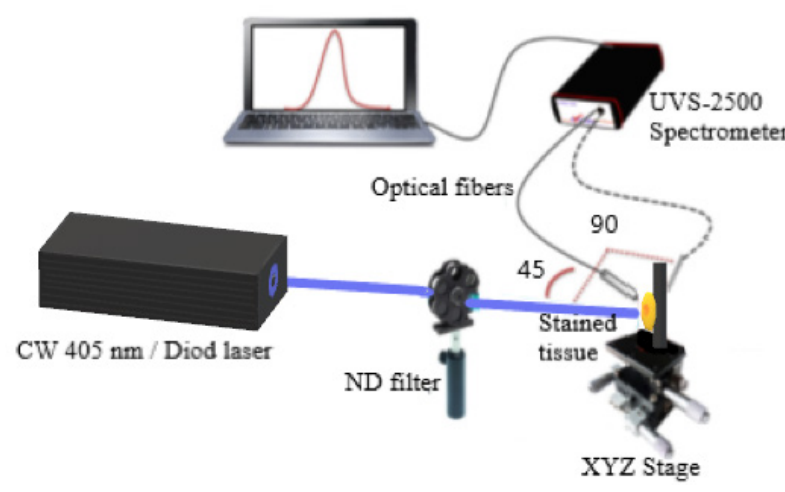

Figure 1. The Schematic of LIF Set-up for the NaFI-Stained Tissues.

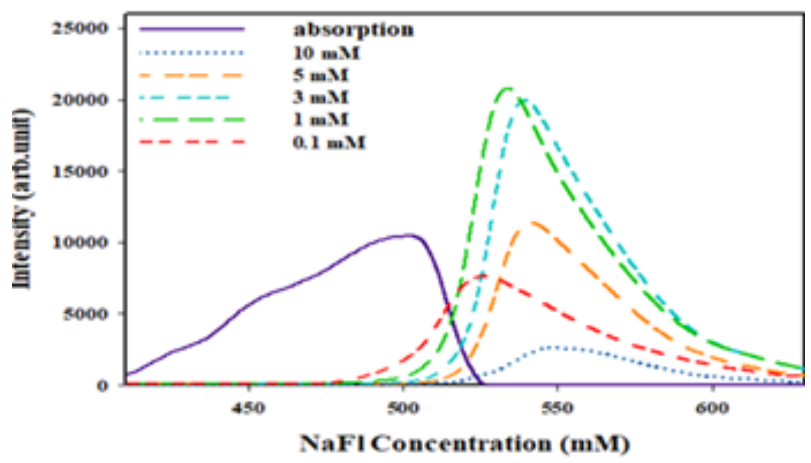

Figure 2. The LIF Emission Spectrum of NaFI Dye at Various Concentrations of 0.1 up to $10 \mathrm{mM}$ of Solvent. 
specimen. Figure 3 illustrates the variation of intensity and the spectral red-shift $(\Delta)$ of emission relative to the absorption versus the $\mathrm{NaFl}$ solution concentration.

According to the results, the cut samples of healthy and cancerous breast tissues (Invasive Ductal Carcinoma) were placed in a $1 \mathrm{mM}$ concentration of dye solution for three hours. After that, the $\mathrm{NaFl}$ infiltrated tissues were irradiated by a laser to record the LIF emission. Figure 4 represents the typical emission spectra from normal and abnormal tissues taken from a patient. It shows that the cancerous tissues exhibit the spectral shift of $8.55 \mathrm{~nm}$ and increased intensity relative to the healthy samples.

Similar experiments for different breast tissue specimens were carried out to examine the repeatability of spectral displacement and increasing the fluorescence intensity of cancerous tissues relative to the healthy ones. Figure 5 illustrates the obtained results to emphasize the red-shift as well as the increased intensity of fluorescence emission of malignant samples.

The biological tissues are highly scattering (opaque) environments. ${ }^{16}$ Then, the multiple scattering of emitted photons by entering a tissue dispersal environment results in the increasing re-absorption and subsequently red-shift in the irregular and disturbed tumor tissues. Moreover, with abnormal cell proliferation, numerous micro-cavities have formed, resulting from structural changes in tubular architecture. ${ }^{17}$ As Figure 6 illustrates, tubular structures are present in the breast tissues, which increases the density and irregularities of the tubules with the increasing tissue malignancy, which means the presence of porosity in tissue..$^{9,12}$

The fluorescence radiation spectrum depends on the external factors (such as $\mathrm{NaFl}$ ) and tissue structure, as a scaffold for fluorophores particles as well as the dye concentration. Therefore, we have also investigated the effect of solution concentration on the spectral redshift. The healthy and cancerous breast tissue samples were placed in a solution of $\mathrm{NaFl}$ in ethanol solvent at concentrations of $0.1,1,3$, and $10 \mathrm{mM}$ for 3 hours

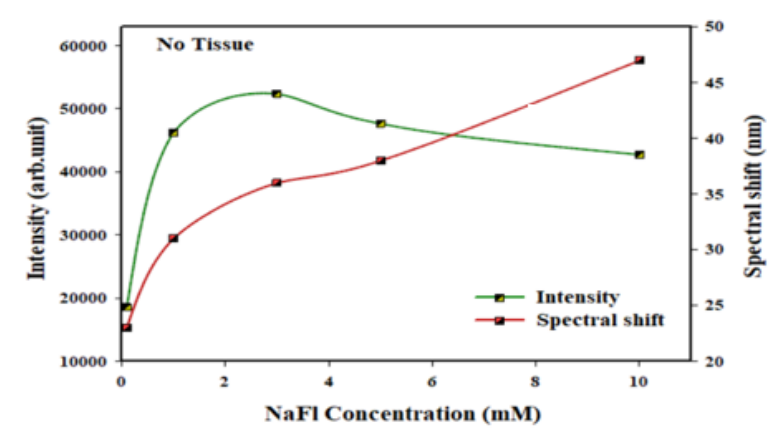

Figure 3. The Intensity (Left Axis) and the Spectral Shift (Right Axis) Between the Absorption and the Emission Spectra at Various Concentrations of NaFI Dye.

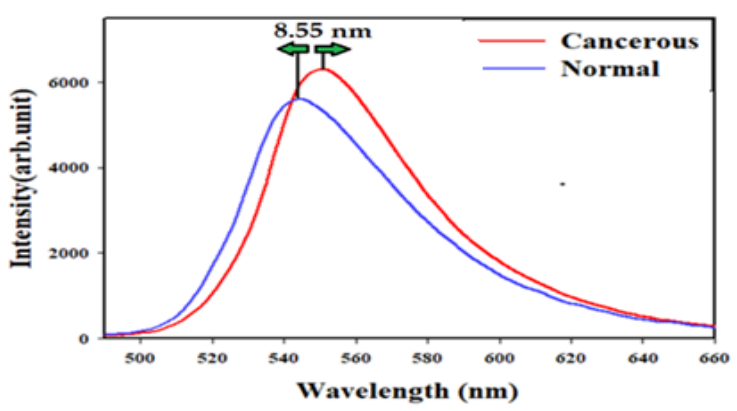

Figure 4. The Typical LIF Spectra Recorded From Normal and Abnormal Breast Samples

at a $1 \mathrm{mM}$ Concentration of $\mathrm{NaFl}$.
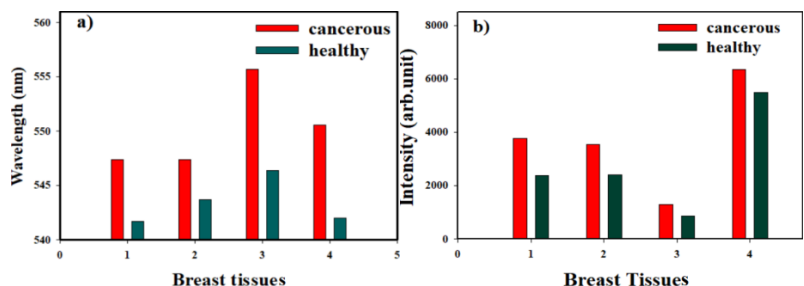

Figure 5. The Wavelength of Fluorescence Peak (a) and the Emission Intensity (b) Taken From Four Cancerous Breast Samples Compared to Their Healthy Ones, at $1 \mathrm{mM}$ Dye Concentration.
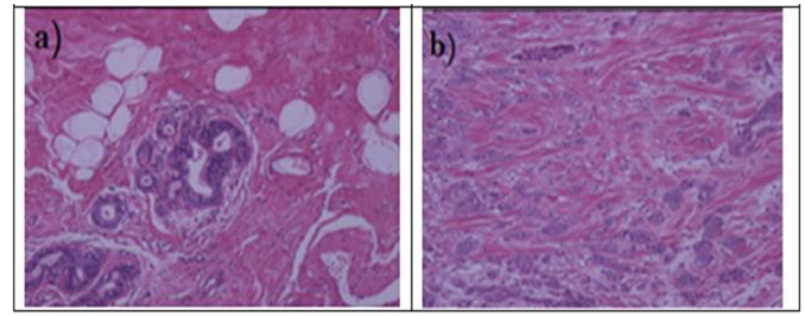

Figure 6. Histological Images of (a) Dye-Stained Normal and (b) Dye-Stained Malignant (Invasive Ductal Carcinoma) Breast Tissues.

and then exposed to radiation. The spectral red-shift increases with increasing the dye concentration for healthy and cancerous tissues, as shown in Figure 7. There is a maximum spectral displacement of $\sim 10.6 \mathrm{~nm}$ in malignant breast tissues compared to healthy ones, at ten $\mathrm{mM}$. In general, the spectral red-shift in malignant tissues results from a high rate of re-absorption.

As noted, cancerous breast tissues have a higher density of tubular structures than healthy tissues, which leads to the capture of dye molecules. The tendency of the dye molecule accumulation in the cancerous tissues is mainly due to the high permeability of the damaged cell membranes with respect to the healthy ones. ${ }^{5}$ Therefore, LIF spectra represent a significant red-shift, and the relative shift of cancerous tumors with respect to healthy ones enhances dye concentration. The spectral shift accounts for the high re-absorption rates in a hybrid 
medium due to the fact that the higher natural abundance of scatterers in the cancerous tissue medium results in the long random walk of scattered photons. Moreover, a large Forster constant $\left(K_{t}\right)$ and the type of chemical bond formed between tissue and the dye molecules, leading to high re-absorption and eventually the sensible spectral shift. ${ }^{5,12,14}$

It is worth noting that by repeating the LIF experiments on several samples of colon and thyroid tissues, similar results were obtained to emphasize the LIF as a method to diagnose cancerous tissues. Figure 8 depicts the typical fluorescence emission recorded from normal and abnormal colon and thyroid samples at $1 \mathrm{mM}$ concentration of $\mathrm{NaFl}$ solution. The specimens of each tissue were taken from one patient. Thus, the pathological (type of malignancy) and even the physical (color) features of healthy and cancerous specimens for each tissue were completely identical. Both LIF signals are more intense and move to the longer wavelengths, which is a marker of the distinction between normal and abnormal tissues and the detection of cancer.

Therefore, as shown in Figure 8, a notable red-shift of 9.1 and $10.2 \mathrm{~nm}$ took place in the malignant colon and thyroid tissues compared to the healthy tissues.

The colon tissues were Adenocarcinoma type with the tubular structure. ${ }^{18,19}$ Therefore, similar to breast tissue samples, it can be stated that the density and irregularity of tubules have increased in cancerous colon tissues compared to healthy specimens (Figure 9).

As shown in Figure 10, this difference in the tubular structure of Adenocarcinoma tissues compared to healthy ones increased the intensity and red-shift for their spectra.

Also, as the type of thyroid cancer tissues in this paper was papillary thyroid carcinoma, therefore, according to Figure 11, the space between the papillae in the cancerous tissues increased compared to the normal ones. Given that the density of the space between the papillae is different from that of the cell regions, colloidal matter is located in this space, which contains thyroid hormones. Given

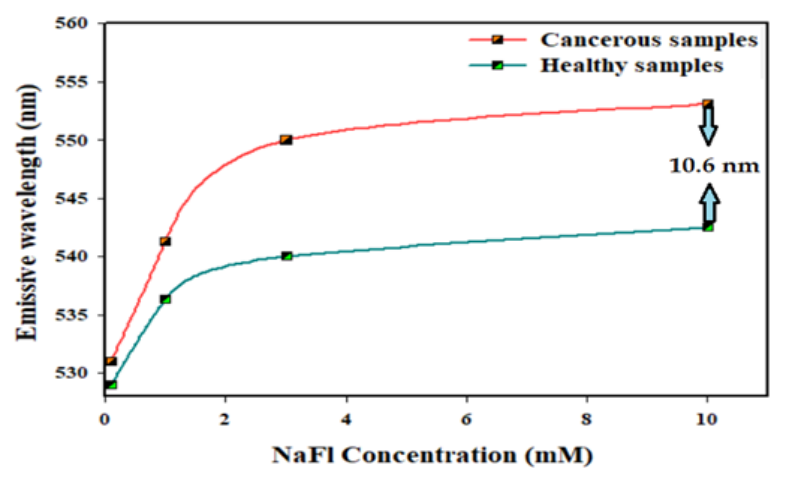

Figure 7. The Spectral Displacement of Stained Healthy and Cancerous Breast Tissues With Different Concentrations (0.1, 1, 3 , and $10 \mathrm{mM}$ ) of $\mathrm{NaFl}$ Solutions.
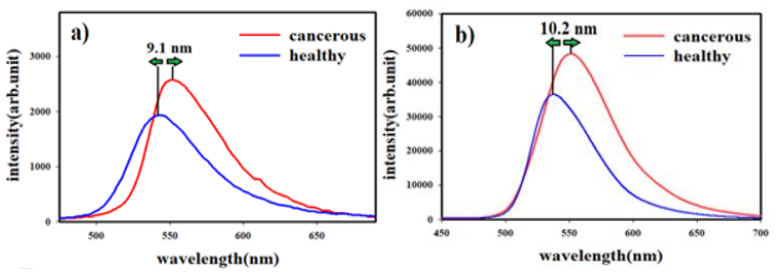

Figure 8. The Typical LIF Spectra Recorded From Normal and Abnormal:

(a) Colon and (b) Thyroid Samples at a $1 \mathrm{mM}$ Concentration of $\mathrm{NaFl}$.
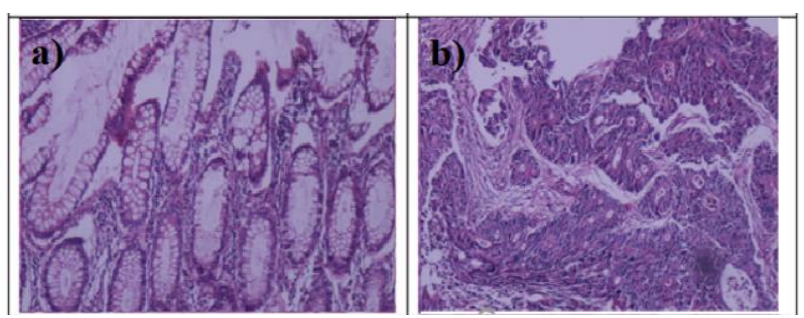

Figure 9. Histological Images of (a) Normal Dye-Stained and (b) Malignant Dye-Stained Colon Tissues (Adenocarcinoma).
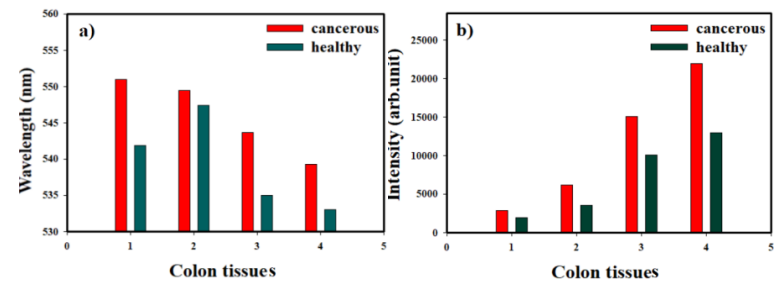

Figure 10. The Wavelength (a) and the Intensity (b) of Fluorescence Peak Taken From Four Cancerous Colon Samples Compared to Their Healthy Ones, at $1 \mathrm{mM}$ Dye Concentration.

that the space between the papillae is completely different from the cellular areas in terms of density and it contains the colloidal material, which contains thyroid hormones and also lacks cells.

The space between the papillae in thyroid samples plays a similar role in tubular structures in breast and colon tissues. Therefore, increasing this space in papillary thyroid carcinoma tissues compared to healthy specimens can cause the red-shift and increase the intensity of the fluorescence spectrum for these cancerous tissues, as shown in Figure 12.

\section{Discussion}

The LIF spectrum taken from stained-NaFl tissues contains valuable information to distinguish between normal and abnormal samples based on the accumulation and behavior of a body-compatible non-toxicity fluorophore.

The micro-partitioning structure of the cancerous tissues decelerates the rate of aggregation processes such that the micro-cavities do trap further fluorophore molecules. 


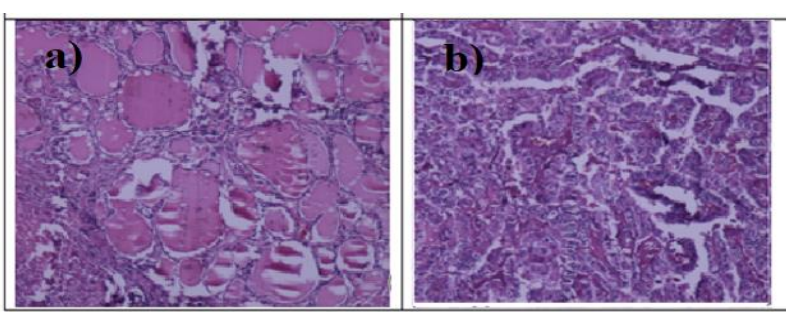

Figure 11. Histological Images of (a) Dye-Stained Normal and (b) Malignant (Papillary Thyroid Carcinoma) Tissues.

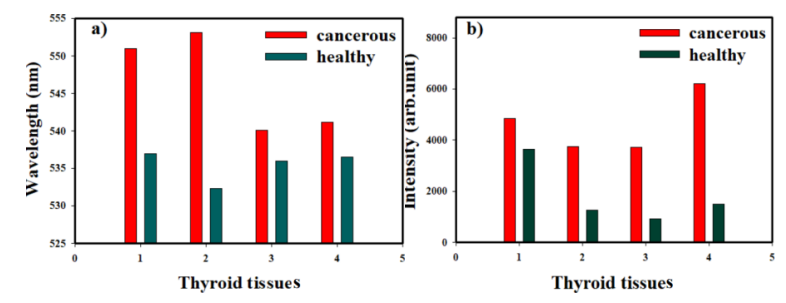

Figure 12. The Wavelength (a) and the Intensity (b) of Fluorescence Peak Taken From Four Cancerous Thyroid Tissues Compared to Their Healthy Ones, at $1 \mathrm{mM}$ Dye Concentration.

This indicates the notable reduction of the self-quenching events. Therefore, by capturing fluorophore molecules, they cause increasing the distance between them and ultimately reduce aggregation and suppression. It leads to an increased fluorescence radiation intensity in cancerous tissues compared with the healthy ones., ${ }^{9,14}$ These results agree with previous reports ${ }^{5,9}$ about increasing the emission intensity and spectral displacement of stainedRd6G cancerous tumor samples relative to healthy tissues.

Also, it is worth mentioning that the used samples in this work are epithelial tissues whose basement membrane is under the epithelial cells. The fluorophore particles penetrate the basal-lateral tissue membrane due to the specific types of connections between the epithelial cells. Here, the entry and accumulation of dye into space between cells, tubules in the colon, and breast tissues, as well as the space between the papillae in the thyroid tissue, were relevant.

The velocity of the dye particles and their penetration into biological structures are different depending on the size and polarity of a permeable fluorophore. ${ }^{21}$ The penetration rate of smaller molecules such as Rhd6G is higher for mono-cationic chromophores with smaller species sizes, such as Rd6G, and the penetration time within the tissue is, on average, 30-45 minutes. ${ }^{9,22}$ However, for the larger dicationic compounds, such as $\mathrm{NaFl}$, the penetration rate is lower, and consequently, it has a longer penetration time (approximately 3 hours). This expression reflects the differences between the interaction of rhodamine and fluorescein with the cells.

\section{Conclusion}

Here, we have assessed the spectral shift and intensity difference of fluorescence as a diagnostic method to distinguish between cancerous and healthy tissues for clinical applications. The least invasive LIF method can be developed with the optimal dose of $\mathrm{NaFl}$ solution without the need for surgery or biopsy for an in-vivo detection of tumor samples. What distinguishes this research is the use of $\mathrm{NaFl}$ as a body-compatible dye at the optimal dosages, rather than toxic ones such as Rhd6G.

\section{Ethical Considerations}

Written informed consent was obtained from the patients before the experiments.

\section{Conflict of Interests}

The authors declare no conflict of interest.

\section{References}

1. Hosseini Motlagh NS, Parvin P, Ghasemi F, Atyabi F. Fluorescence properties of several chemotherapy drugs: doxorubicin, paclitaxel, and bleomycin. Biomed Opt Express. 2016;7(6):2400-2406. doi: 10.1364/BOE.7.002400.

2. Surmacki J, Musial J, Kordek R, Abramczyk H. Raman imaging at biological interfaces: applications in breast cancer diagnosis. Mol Cancer. 2013;12(1):48. doi: 10.1186/1476-4598-12-48.

3. Ghasemi F, Parvin P, Hosseini Motlagh NS, Amjadi A, Abachi S. Laser-induced breakdown spectroscopy and acoustic response techniques to discriminate healthy and cancerous breast tissues. Appl Opt. 2016;55(29):8227-8235. doi: 10.1364/AO.55.008227.

4. Ramanujam N. Fluorescence spectroscopy of neoplastic and non-neoplastic tissues. Neoplasia. 2000;2(1-2):89-117. doi: 10.1038/sj.neo.7900077.

5. Ghasemi F, Parvin P, Hosseini Motlagh NS, Keraji M, Hadavand Mirzaee F, Bavali A. Optical spectroscopic methods to discriminate in-vitro Hodgkin cancerous and normal tissues. Int J Opt Photonics. 2015;9(2):65-72. doi: 10.1364/BODA.2015.JT3A.40.

6. Salman M, Mohammad Hosein MA, Mohammad N. Nonlinear optical investigation of normal ovarian cells of animal and cancerous ovarian cells of human invitro. Optik. 2016;127(8):3867-3870. doi: 10.1016/j. ijleo.2016.01.072.

7. Palmer GM, Keely PJ, Breslin TM, Ramanujam N. Autofluorescence spectroscopy of normal and malignant human breast cell lines. Photochem Photobiol. 2003;78(5):462-9. doi: 10.1562/0031-8655(2003)078<0462:asonam>2.0.co;2.

8. Galmed AH, Elshemey WM. The diagnostic capability of laser-induced fluorescence in the characterization of excised breast tissues. Laser Phys. 2017;27(8):085601. doi:10.1088/1555-6611/aa7ce4.

9. Ghasemi F, Parvin P, Hosseini Motlagh NS, Abachi S. LIF spectroscopy of stained malignant breast tissues. Biomed Opt Express. 2017;8(2):512-523. doi: 10.1364/ BOE.8.000512.

10. Braginskaja OV, Lazarev VV, Polsachev VI, Rubin LB, 
Stoskii VE. Fluorescent diagnostics of human gastric cancer and sodium fluorescein accumulation in experimental gastric cancer in rats. Cancer Lett. 1993;69(2):117-21. doi: 10.1016/0304-3835(93)90163-4.

11. Lalayan AA, Aydinyan L, Harutyunyan I, Galstian HM. Multifrequency YAG:Nd laser for diagnosis of malignant tumors. Proceedings of the SPIE, Optical Biopsies and Microscopic Techniques III; 1998 Jan; 3414: 11-16. doi: 10.1117/12.336817.

12. Ghasemi F, Parvin P, Lotfi M. Laser induced fluorescence spectroscopy for diagnosis of cancerous tissue based on the fluorescence properties of formaldehyde. Laser Phys Lett. 2019;16(3):035601. doi: 10.1088/1612-202X/AAF89D.

13. Lakowicz JR. Principles of Fluorescence Spectroscopy. $3^{\text {rd }}$ ed. New York: Springer; 2006. doi: 10.1007/978-0-387-46312-4 .

14. Bavali A, Parvin P, Mortazavi SZ, Mohammadian M, Mousavi Pour MR. Red/blue spectral shifts of laser-induced fluorescence emission due to different nanoparticle suspensions in various dye solutions. App Opt. 2014;53(24):5398-5409. doi: 10.1364/AO.53.005398.

15. Hosseini Motlagh NS, Parvin P, Ghasemi F, Atyabi F, Jelvani S, Abolhosseini S. Laser induced fluorescence spectroscopy of chemo-drugs as biocompatible fluorophores: irinotecan, gemcitabine and navelbine. Laser Phys Letters.
2016,13(7):075604. doi: 10.1088/1612-2011/13/7/075604.

16. Perelman LT. Optical diagnostic technology based on light scattering spectroscopy for early cancer detection. Expert Rev Med Devices. 2006;3(6):787-803. doi: 10.1586/17434440.3.6.787.

17. Polson RC, Vardeny ZV. Random lasing in human tissues. Appl Phys Lett. 2004;85(7):1289-91. doi: 10.1063/1.1782259.

18. Zonios GI, Cothren RM, Arendt JT, Wu J, Van Dam J, Crawford JM, et al. Morphological model of human colon tissue fluorescence. IEEE Trans Biomed Eng. 1996;43(2):113-22. doi: 10.1109/10.481980.

19. Odze RD, Goldblum JR. Odze and Goldbum Surgical Pathology of the GI Tract, Liver, Biliary Tract, and Pancreas. 3rd ed. Philadelphia : Saunders Elsevier ; 2014.

20. Kumar V, Abbas A, Fausto N, Aster J. Robbins and Cotran Pathologic Basis of Disease. 8th ed. Philadelphia: Saunders Elsevier; 2009.

21. Stillwell W. An introduction to biological membranes: composition, structure and function. 2nd ed. Amsterdam: Elsevier Science; 2016.

22. Mogharari N, Sajad B. Random laser emission spectra of the normal and cancerous thyroid tissues. Iran J Sci Technol Trans A Sci. 2019;43:2055-2060. doi: 10.1007/s40995-01900691-8. 